\title{
USO DEL PLASMA RICO EN PLAQUETAS AUTÓLOGO EN ABDOMINOPLASTÍAS*
}

\author{
Drs. María Luisa García-García ${ }^{1}$, Ramón José Lirón-Ruiz \\ Juan Gervasio Martín-Lorenzo ${ }^{1}$, José Antonio Torralba-Martínez ${ }^{1}$, \\ María Ángeles Verdú-Fernández ${ }^{1}$, José Luis Aguayo-Albasini ${ }^{1}$
}

1 Servicios de Digestivo y Cirugía General del Hospital General Universitario “JM Morales Meseguer”. Campus de Excelencia Internacional "Mare Nostrum". Universidad de Murcia.

Murcia, España.

\begin{abstract}
\section{Using platelet rich plasma in abdominoplasty}

Objective: Evaluation of the effectiveness of autologous platelet rich plasma administered topically in the healing and prevention of complications of abdominoplasty after bariatric surgery. Material and Methods: The data from 30 patients who underwent abdominoplasty were analyzed: Group I: Rich plasma was administered autologous platelets in the surgery. Group II: Control. Study variables: complications (seroma, local infection and bleeding), length of stay and cost. Results: No significant differences were observed in the frequency of complications or length of stay. The use of platelet rich plasma raises the cost of the procedure. Conclusion: Even considering the limitations of the study because of its small sample size and lack of blinding, the results do not support the use of this complementary technique. Further studies are needed in the field of adhesives, sealants and local hemostatic.
\end{abstract}

Key words: Platelet rich plasma, abdominoplasty, complication.

\section{Resumen}

Objetivo: Evaluación de la eficacia del plasma rico en plaquetas autólogo administrado de forma tópica en la cicatrización y prevención de las complicaciones de la abdominoplastía y dermolipectomía tras cirugía bariátrica. Material y Método: Serie de 30 pacientes afectos de faldón abdominal tras cirugía bariátrica, a los que se practicó dermolipectomía y abdominoplastía, divididos en dos grupos de 15 casos con aletorización simple. Grupo I: Se administró plasma rico en plaquetas autólogo en el lecho quirúrgico. Grupo II: Control. Variables de estudio: complicaciones (seroma, infección local y hemorragia), tiempo de estancia y coste. Resultados: No se aprecian diferencias significativas en la frecuencia de complicaciones ni en estancia media. El uso de plasma rico en plaquetas eleva el coste del procedimiento. Conclusión: Aun considerando las

*Recibido el 8 de septiembre de 2014 y aceptado para publicación el 30 de marzo de 2015.

Los autores no refieren conflictos de interés.

Correspondencia: Dra. María Luisa García-García

mlgrgr@gmail.com 
limitaciones del estudio por su corto número de casos y no cegamiento, los resultados no apoyan el uso de esta técnica complementaria. Estudios más avanzados son necesarios en el campo de los adhesivos, sellantes y hemostáticos locales.

Palabras clave: Plasma rico en plaquetas, abdominoplastía, complicaciones.

\section{Introducción}

La dermolipectomía y abdominoplastía es un proceso quirúrgico cada vez más frecuente, con más de 100 años de experiencia desde que Kelly ${ }^{1}$ lo describiera en 1899. Por otra parte, después de la cirugía bariátrica, hay pacientes que buscan una solución al exceso de piel y tejido subcutáneo abdominal que queda tras una pérdida masiva de peso. En la literatura existen distintos estudios que analizan las complicaciones asociadas a estos procedimientos y hay una gran controversia sobre los factores de riesgo asociados y la posibilidad de prevenir dichas secuelas ${ }^{2}$. La gran extensión en la disección de los tejidos produce como complicación más frecuente el seroma, cuya incidencia presenta un rango variable de $0,3-90 \% \%^{3}$.

El plasma rico en plaquetas autólogo es un producto derivado de la sangre del propio paciente cuya aplicación en la práctica clínica es cada vez mayor. Su uso está muy extendido en diversas especialidades tanto en la curación de heridas quirúrgicas complejas como en la regeneración de tejidos perdidos ${ }^{3}$.

Presentamos nuestra experiencia en el uso de plasma autólogo rico en plaquetas (PRP) con el fin de evaluar su eficacia en la cicatrización y prevención de las complicaciones de la abdominoplastía y dermolipectomía.

\section{Material y Método}

\section{Pacientes}

La serie está constituida por 30 pacientes ( 9 varones y 21 mujeres, con edad media 43,03 $\pm 10,77$ ) a los que se les realizó dermolipectomía y abdominoplastía, después de una gran pérdida de peso tras cirugía bariátrica, en el Hospital Universitario José María Morales Meseguer. El estudio abarca un período de 1 año.

Estudio prospectivo randomizado simple. Grupos de estudio: a) Grupo I o PRP: En 15 pacientes se usó PRP (extraído mediante centrifugado de la sangre recogida del paciente) sobre la aponeurosis; b) Grupo II o NO PRP: En los otros 15 se realizó la técnica quirúrgica sin empleo del producto.

Se recogieron: Datos demográficos como edad y sexo, índice de masa corporal (IMC) antes de la cirugía bariátrica, IMC previo a la abdomino- plastía, tiempo entre cirugías, las co-morbilidades como hipertensión arterial, diabetes mellitus, y hábito tabáquico. Se registró el peso de la pieza de dermolipectomía. Las variables de estudio fueron: formación de seroma, infección del sitio quirúrgico, hematoma o sangrado, tasa de reintervención y estancia media. Se compararon las variables en ambos grupos. El estudio fue aprobado por el Comité de Etica de nuestro hospital.

\section{Descripción de la técnica quirúrgica}

La dermolipectomía comienza con una incisión en el límite superior del triángulo piloso pubiano que se dirige horizontalmente hacia ambos lados hasta las espinas ilíacas anterosuperiores. Se realiza el despegamiento supraaponeurótico en sentido craneal del labio superior. El gran colgajo se levanta y se secciona en la línea media hasta el ombligo donde, mediante una incisión circular, se separa del colgajo abdominal. Se continúa hasta alcanzar el apéndice xifoides y los rebordes costocondrales. Se inclina la mesa operatoria en ángulo de $15^{\circ}$ en posición anti Trendelenburg, colocando una sutura temporal en la línea media para fijar el colgajo. El asistente tira de cada aleta del colgajo superior caudalmente, estimando el exceso de tejido sobrante a resecar. Se marca la posición para exteriorizar el ombligo, previo a la sutura final. Se exterioriza el ombligo mediante la ayuda de puntos de sutura largos y se fija a la piel vecina con puntos finos separados. Se colocan dos drenajes aspirativos y se lleva a cabo el cierre del plano subcutáneo y de la piel.

Se empleó profilaxis antibiótica con cefalosporinas de $2^{\mathrm{a}}$ generación, y antitromboembólica con heparina de bajo peso molecular, en todos los casos.

\section{Obtención de plasma rico en plaquetas}

El plasma se aísla de la sangre recogida del paciente tras la anestesia. Se preparan cuatro tubos estériles de 4,5 $\mathrm{ml}$ con citrato sódico 3,8\% como anticoagulante. La sangre se procesa inmediatamente, centrifugándola a 300-400 G durante 7-8 min a temperatura ambiente, mediante el sistema gravitacional de separación de plaquetas GPS ${ }^{\circledR}$ III (Concentrador de plasma Plasmax Plus, Biomet Biologics, Warshaw, Indiana, USA). El plasma obtenido se separa en fracciones mediante pipeteo meticuloso: el tercio superior se denomina plasma pobre en factores de crecimiento y ocupa alrededor de $0,5 \mathrm{ml}$. En el tercio medio, se encuentra el plasma con una 
Tabla 1. Variables sociodemográficas, co-morbilidades y características quirúrgicas y estancia media

\begin{tabular}{|lccc|}
\hline Variables & Grupo PRP & Grupo no PRP & p \\
\hline Edad (años) & $44 \pm 11,6$ & $42 \pm 10,1$ & 0,744 \\
HTA & 5 & 7 & 0,456 \\
DM & 5 & 2 & 0,390 \\
Fumador & 5 & 5 & 1 \\
IMC $\left(\mathrm{kg} / \mathrm{m}^{2}\right.$ ) previo a cirugía bariátrica & $48,5 \pm 7.8$ & $49,9 \pm 2,5$ & 0,935 \\
IMC $\left(\mathrm{kg} / \mathrm{m}^{2}\right)$ previo a la abdominoplastía & $26,1 \pm 2.5$ & $27,2 \pm 5,5$ & 0,967 \\
Tiempo medio entre cirugías (meses) & $28,1 \pm 19,2$ & $30 \pm 19,4$ & 0,305 \\
\hline Peso medio de la pieza (kg) & $1.829 \pm 749,6$ & $2.225 \pm 960$ & 0,285 \\
\hline Estancia media (días) & $4,1 \pm 1.5$ & $4,8 \pm 1,5$ & 0,267 \\
\hline
\end{tabular}

IMC: Índice de masa corporal. HTA: Hipertensión arterial. DM: Diabetes Mellitus.

concentración media de factores de crecimiento y ocupa también $0,5 \mathrm{ml}$. El tercio inferior que queda inmediatamente por encima del concentrado de hematíes se conoce como plasma rico en factores de crecimiento o plasma autólogo rico en plaquetas (PRP) con el mismo volumen. Posteriormente se procede a la activación del PRP mediante cloruro cálcico al $10 \%(0,05$ de cloruro cálcico por cada $\mathrm{ml}$ de PRP). Obtenemos así un suero amarillento de consistencia líquida. Si se quisiera textura gel se tendría que añadir más citrato. El producto, en el Grupo I, se administró mediante rociado sobre la aponeurosis.

El coste del sistema y aplicación del Concentrador de plasma Plasmax Plus es de 403 Euros por paciente.

\section{Análisis estadístico}

La comparación entre variables cualitativas se ha efectuado mediante la aplicación de la prueba de $\chi^{2}$ de Pearson, $\chi^{2}$ de tendencia lineal y exacta de Fisher. La comparación entre variables cuantitativas y una cualitativa de dos opciones se ha analizado mediante la prueba $t$ de Student si la variable cuantitativa se distribuye de forma normal, y mediante la prueba de Mann Whitney si la distribución es no paramétrica. Se ha utilizado el programa informático SPSS versión 15.0 para Windows (SPSS; Chicago, IL).

\section{Resultados}

Ambos grupos fueron homogéneos en variables socio-demográficas, patologías asociadas e IMC (Tabla 1). Se apreciaron complicaciones en 7 pacientes $(46,7 \%)$ del grupo PRP, y en 8 pacientes $(53,3 \%)$ del grupo NO PRP, sin diferencias significativas. La secuela más frecuente fue el seroma, requiriendo punción evacuadora en todos los casos. Presentó una incidencia del $40 \%$ en el grupo de PRP frente a un $26,7 \%$ del grupo NO PRP. Comparando ambos grupos no se encontró diferencias significativas ( $p$ $=0,439$ ). Dos pacientes del grupo I tuvieron una infección de herida frente a 3 pacientes del grupo II, un paciente de este último grupo requirió desbridamiento quirúrgico y antibiótico intravenoso. El resto se solucionó con curas locales y antibiótico vía oral. Respecto a la infección, no se hallaron diferencias significativas. En cuanto a la incidencia de sangrado o hematoma, en el grupo en el que se usó el producto, un paciente sufrió un sangrado que requirió cirugía urgente, y 5 pacientes del grupo sin producto presentaron sangrado o hematoma (dos de ellos necesitaron transfusión sanguínea y uno cirugía urgente). Tampoco se encontraron diferencias estadísticamente significativas (Tabla 2).

No hubo diferencias en cuanto a la tasa de reintervención. La estancia media en grupo PRP fue de $4,1 \pm 1,5$ días, ligeramente inferior al grupo NO PRP con $4,8 \pm 1,5$ días $(\mathrm{p}=0,267)$.

Tabla 2. Complicaciones postoperatorias

\begin{tabular}{|lccc|}
\hline Variables & $\begin{array}{c}\text { Grupo } \\
\text { PRP }\end{array}$ & $\begin{array}{c}\text { Grupo } \\
\text { no PRP }\end{array}$ & p \\
\hline $\begin{array}{l}\text { Pacientes que sufrieron } \\
\text { alguna complicación }\end{array}$ & 8 & 7 & 0,715 \\
Seroma & 6 & 4 & 0,439 \\
Infección & 2 & 3 & 1 \\
Sangrado & 3 & 1 & 0,598 \\
Hematoma & 2 & 0 & 0,483 \\
Reintervención & 1 & 2 & 1 \\
\hline
\end{tabular}




\section{Discusión}

La complicación más frecuente de la dermolipectomía y abdominoplastía es el seroma. Así surge de la necesidad de reducir su tasa, sobre todo en aquellos casos con grandes despegamientos cutáneos $^{3-5}$. Con los últimos avances tecnológicos, aparecen en el mercado los nuevos sellantes biológicos de los que hay pocas publicaciones acerca de su eficacia.

El PRP se define como una fracción de plasma obtenido de sangre autóloga que tiene una concentración de plaquetas superior a la del plasma en condiciones basales, contiene no sólo un alto nivel de plaquetas, sino también de los factores de crecimiento que son secretados activamente por las plaquetas. Su principal ventaja es la utilización de sangre del propio paciente, por lo que su aplicación en la práctica clínica es cada vez mayor ${ }^{6}$.

Sus predecesores fueron el adhesivo de fibrina (alógeno o xenógeno), utilizado como hemostático y adhesivo quirúrgico, y el fibrinógeno autólogo, con el que se preparaba el gel de fibrina autóloga, que requería ingreso del paciente días antes de la intervención para su extracción y procesamiento. El plasma rico en plaquetas se presenta así como una buena opción frente a estos adhesivos industriales, que tienen propiedades similares de adhesión y hemostasia, pero con mayores costes y también el riesgo, aunque pequeño, de la transmisión viral y reacciones alérgicas ${ }^{3,7}$.

La gran popularización del PRP tuvo sus orígenes en los campos de la cirugía oral y la odontología por los efectos estimulantes de este preparado sobre los fibroblastos de la estructura periodontal y la mucosa oral, y sobre los osteblastos del hueso alveolar ${ }^{8}$. Su uso está muy extendido además en otras especialidades por favorecer la curación de heridas quirúrgicas y regenerar tejidos perdidos. El PRP es rico en proteínas que actúan a nivel de la adhesión celular (fibrina, fibronectina, y vitronectina), por lo que proporciona el soporte estructural necesario para la migración celular, y para la proliferación y crecimiento tridimensional de los tejidos sobre los que actúa. Actúa no sólo directamente sobre las células diana para los factores de crecimiento, sino también como matriz extracelular para la estimulación de la reparación y/o regeneración del tejido de un modo global. Además se considera que reduce la inflamación postoperatoria y la acumulación de líquido, hematoma y seroma debido a las propiedades hemostáticas y antigénicas derivadas de los factores de crecimiento de plaquetas ${ }^{8-10}$. En el PRP se aíslan: factor de crecimiento de origen plaquetario (PDGF), factor de crecimiento fibroblástico (FGF), factor de crecimiento similar a la insulina (IGF), factor de crecimiento epidérmico (EGF) y factor de transformación beta ( TGF- $\beta$ ) tipos 1 y $2^{5,6-10}$.

Por lo que, la presencia de PRP acelera la cicatrización imitando el proceso natural de curación de heridas: las plaquetas forman un coágulo inicialmente y segregan diversas citoquinas que inducen respuestas celulares de quimiotaxia, angiogénesis, síntesis de material extracelular, etc.

Además, se ha utilizado para cohesionar injertos óseos o biomateriales articulados, como membrana biológica o en forma de spray para aumentar la adhesividad de colgajos cutáneos o mucosos al lecho receptor. Por sus efectos sobre los tejidos blandos, el PRP se ha usado en cirugía plástica y dermatológica para favorecer y modular la curación en los colgajos cutáneos repuestos, ofreciendo una mejor regeneración tisular, un mejor sellado y una mejor hemostasia de todos los procedimientos quirúrgicos que llevan implícito el levantamiento de un colgajo ${ }^{8}$. Sin embargo, hay pocas publicaciones sobre uso de plasma rico en plaquetas en cirugía, particularmente autólogo, con resultados dispares. En los estudios de Schettino et al. ${ }^{3}$ y Llewwllyn-Bennett et al. ${ }^{12}$, no observan una disminución de la tasa de seroma cuando se usa un sellante de fibrina al igual que nuestro estudio. En contraposición al estudio de Jackson $^{5}$, que encuentra los beneficios de su uso en la mejoría de la cicatrización y la disminución de la incidencia de seroma.

En cuanto a nuestro estudio comparativo entre pacientes con uso del producto y sin producto, las tasas de infección y sangrado/hematoma fueron menores en el grupo en el que se usó el producto, pero no se encontró relación estadística significativa. Tampoco en cuanto a la tasa de reintervención y la estancia media en el grupo PRP fue ligeramente inferior al grupo NO PRP. Tampoco se encontraron diferencias en cuanto a la tasa de seroma.

No obstante, tras observar la alta tasa de seroma de nuestra serie, se han propuesto distintas estrategias de prevención: acortar tiempos quirúrgicos para disminuir la hipotermia y la excesiva exposición de tejido, evitar extirpaciones amplias que aumentan el riesgo de sangrado e infección, limitando el despegamiento superior o cirugía en dos tiempos, uso del bisturí armónico, para mejorar la coagulación y sellado de vasos o nuevos pegamentos para prevención de seromas o hematomas ${ }^{2}$.

Aunque los resultados de nuestra investigación son preliminares y necesitan mayor estudio (entre las limitaciones de nuestro trabajo: no cegamiento y pequeño tamaño muestral), el uso de plasma autólogo no parece aportar beneficios en la reducción de las complicaciones postoperatorias en las abdominoplastías y, en su contra, aumenta el coste del procedimiento. 


\section{Referencias}

1. Kelly HA. Report of gynaecological cases. Johns Hopkins Med J. 1899;10:197.

2. García-García ML, Martín-Lorenzo JG, Campillo-Soto A, Torralba-Martínez JA, Lirón-Ruiz R, Miguel-Perelló $\mathrm{J}$, et al. Complications and level of satisfaction after dermolipectomy and abdominoplasty post-bariatric surgery. Cir Esp. 2014;92:254-60.

3. Schettino AM, Franco D, Franco T, Filho JM, Vendramin FS. Use of autologous fibrin glue (platelet-poor plasma) in abdominal dermolipectomies. Aesthetic Plast Surg. 2012;36:1296-301.

4. Franco D, Franco T, Schettino AM, Filho JM, Vendramin FS. Protocol for obtaining platelet-rich plasma (PRP), platelet-poor plasma (PPP), and thrombin for autologous use. Aesthetic Plast Surg. 2012;36:1254-9.

5. Jackson RF. Using platelet-rich plasma to promote healing and prevent seroma formation in abdominoplasty procedures. Am J Cosmet Surg. 2003;20:185-95.

6. Mehta S, Watson JT. Platelet rich concentrate: basic science and current clinical applications. J Orthop Trauma. 2008;22:432-8.

7. Reyes M, Montero S, Cifuentes J, Zarzar E. Actualización en la Técnica de Obtención y Uso de Plasma Rico en Factores de Crecimiento (PRGF). Rev Dent Chil. 2002;93:25-8.

8. Rodríguez-Flores J, Palomar-Gallego MA, Torres García-Denche. Plasma rico en plaquetas: fundamentos biológicos y aplicaciones en cirugía maxilofacial y estética facial. Rev Esp Cir Oral Maxilofac. 2012;34: 8-17.

9. Wu X, Ren J, Luan J, Yao G, Li J. Biochemical, mechanical, and morphological properties of a completely autologous platelet rich wound sealant. Blood Coagul Fibrinolysis 2012;23:290-5.

10. Chandarana S, Fung K, Franklin JH, Kotylak T, Matic DM, Yoo J. Effect of autologous platelet adhesives on dermal fat graft resorption following reconstruction of a superficial paratiroidectomy defect: a double-blinded prospective trial. Head Neck 2009;31:521-30.

11. Burón-Álvarez I, Fernández-Tresguerres A, Calvo M, Alfageme F, Villegas C, Fernández R. Tratamiento de úlceras cutáneas crónicas con plasma autólogo rico en plaquetas. Piel 2012;27:429-34.

12. Llewellyn-Bennett R, Greenwood R, Benson JR, English R, Turner J, Rayter Z, et al. Randomized clinical trial on the effect of fibrin sealant on latissimusdorsidonor- site seroma formation after breast reconstruction. Bristh J Surg. 2012;99:1381- 8. 